\title{
The role of platelets in the pathophysiology of atherosclerosis (Review)
}

\author{
IVÁN PALOMO, CARLA TORO and MARCELO ALARCÓN
}

\begin{abstract}
Research Program of Cardiovascular Disease Risk Factors, Department of Clinical Biochemistry and Immunohematology, Faculty of Health Sciences, University of Talca, Chile
\end{abstract}

Received December 4, 2007; Accepted January 15, 2008

\begin{abstract}
The physiopathology of atherothrombosis is complex. The development and progression of this vascular disease involves the interactive processes of atherosclerotic lesions and the formation of thrombi. In and of itself, atherosclerosis is not deadly; the actual risk lies in the vulnerability of the arteriolosclerotic plaque to breakage. An ulceration, which is the rupture or breakage of the covered plaque, constitutes a complication that favors thrombosis. During these processes platelets are relevant factors, acting with the endothelial and inflammatory cells even at the premature stages of atherogenesis. The interaction of platelets with the endothelial cells (ECs) can occur in two ways: activated platelets joining intact ECs, or resting platelets joining activated ECs. Platelet molecules such as GPIIb-IIIa, CD40, CD40L and P-selectin, as well as microparticle platelets, are important in this process. In this review, the most important mechanisms by which platelets participate in the genesis of atherosclerotic lesions are described.
\end{abstract}

\section{Contents}

1. Introduction

2. Platelet physiology

3. Platelets and atherogenesis

4. Conclusion

\section{Introduction}

Cardiovascular diseases (CVDs) are a heterogeneous group of diseases that affect the circulatory system and heart. According to the World Health Organization, CVDs are the principal cause of mortality worldwide, responsible for

Correspondence to: Dr Iván Palomo, Department of Clinical Biochemistry and Immunohematology, Faculty of Health Sciences, University of Talca, P.O. Box 747, Talca, Chile

E-mail: ipalomo@utalca.cl

Key words: platelets, atherosclerosis, thrombosis, endothelial cells approximately $30 \%$ of global deaths. With time, they are gaining increasing relative importance, due in part to the phenomenon of population aging (1).

Endothelial lesions and platelet activation are the most important mechanisms in the physiopathology of arterial thrombosis, which usually occurs in association with preexistent vascular diseases, namely atherosclerosis. The principal risk factors for arterial thrombosis are arterial hypertension (AHT), smoking, dyslipemia and diabetes (2).

Atherosclerosis is a pathology which occurs due to established factors as a consequence of the proliferate inflammation of the endothelium, classically known as the enlargement of the arterial membranes through the gradual accumulation of cholesterol, calcium and macrophages. As time passes, these deposits become tough and narrow the arterial lumen, which may block blood flow (2).

Atherosclerosis in and of itself is not deadly; the actual risk lies in the vulnerability of the arteriolosclerotic plaque to breakage. An ulceration, the rupture or breakage of the covered plaque, constitutes a complication that favors thrombosis and the subtotal or total sharp coronary occlusion, and causes an acute myocardium infarct (AMI) or encephalic vascular accident (EVA) according to the state of the compromised artery (3).

Although progress has been made in elucidating the cell and molecular mechanisms involved in the pathogenesis of atherothrombosis, there are aspects of the disease which remain to be clarified. In this review, the participation of the platelets in the early stages of atherogenesis is discussed.

\section{Platelet physiology}

Platelets participate not only in primary but also in secondary haemostasis. The process can involve many stages, such as platelet adhesion, platelet aggregation and pro-coagulant activity $(4,5)$.

Platelet adhesion. In response to vascular damage the platelets, which normally circulate in an isolated fashion, instead stick to the subendothelial matrix. This initiates interaction between platelet membrane glycoproteins and adhesive protein from the endothelium, collagen and the Von Willebrand factor (FVW). Under low flow conditions, this interaction is conducted by GPIa-IIa platelet glycoprotein; under high flow conditions, the GPIb-IX-V complex participates (6). 
GPVI (62 kDa) belongs to the immunoglobulin superfamily and, along with GPIa-IIa, acts as a collagen receptor $(4,5)$. On the platelet surface, it forms a complex with the receptor $\mathrm{Fc} \gamma$, activating the phosphorylation of a cytoplasmatic segment of the receptor and producing platelet activation.

The GPIb-IX complex (CD42b, CD42a, CD42d) acts as a receptor for FVW, participating in the platelet adhesion mechanism. Resting platelets express $\sim 25,000$ copies of this glycoprotein complex (6).

Platelet aggregation. Once the platelets have adhered, they assume a rounded form with prolongations that exhibit the GPIIb-IIIa complex, forming dimers of the glycoprotein to join the fibrinogen or FVW, according to the flow, and to release the content of their granules. This activates and adds more platelets (6).

The GPIIb-IIIa complex (integrin $\alpha I I b ß 3$, CD41-CD61) is a $228-\mathrm{kDa}$ heterodimer whose structure presents an extracellular domain as a transmembrane and a small cytoplasmatic domain. Every platelet displays $~ 40,000-50,000$ copies of this glycoprotein complex.

As well as binding fibrinogen and FVW, the GPIIb-IIIa complex can also bind to fibronectin and vitronectin, thus participating in the adhesion and aggregation platelet mechanism (5).

Platelet secretion. Two types of granules are functionally the most important in platelets: $\alpha$ granules and dense granules. $\alpha$ granules store large amounts of proteins, among them fibrinogen, FVW, platelet factor 4 (PF4), GPIIb-IIIa, FV, FVIII, FXI, platelet-derived growth factor (PDGF) and epidermal growth factor (EGF), which participate in platelet aggregation. On the other hand, dense granules are rich in calcium, phosphorus, ADP and ATP; the release of their content has a special importance in the amplification of the platelet response (5).

Pro-coagulant activity. Lipid disposition in the platelet membrane is of functional importance. Two enzymes, a translocase specific to aminophospholipids and a flopase, maintain the asymmetric state of phospholipids in the membrane. During the activation of platelets, a redistribution of anionic phospholipids, mainly phosphatidylserine (PS), occurs from inside to outside by the action of the enzyme scramblase (7), which is activated by the increase in cytosolic calcium of platelets and creates a surface with a negative electric charge (5). This favours the formation of coagulation complexes and, consecutively, the formation of fibrin and the consolidation of the thrombus (6).

In addition to PS expression, platelet activation also contributes to the coagulation process, the release of PF4, FXa and FXIII (6) and, as recently described, the synthesis and expression of tissue factor (TF) upon their move to the site of the lesion (8).

\section{Platelets and atherogenesis}

Over the last few years, it has been demonstrated that platelets do not only participate in thrombus complications of atheromastosus injury, but also in the initiation and progression of atherosclerotic plaques (9). In this sense, platelets represent a bridge joining inflammation and thrombosis, processes fundamental to the development of atherothrombosis.

The interaction between the platelets and the endothelial cells (ECs) occurs in two ways: activated platelets can join intact ECs, or resting platelets can adhere to activated ECs. In addition, interaction between activated platelets and activated ECs can occur.

Activated platelets and intact EC. Platelets play an important role in thrombotic and inflammatory processes (10). The circulation of activated platelets has been shown to be one of the initial causes of atherosclerosis development. This is mediated by the interaction between the GPIIb-IIIa of activated platelets, ICAM-1 in ECs (11) and P-selectin (CD62P) expressed in activated platelets which bind to the endothelium (12).

Animal models have also demonstrated the important role of P-selectin in the development of atherogenesis. Animals which lose P-selectin expression show a decrease in atherosclerotic injury (13).

It has been demonstrated that A disintegrin and metalloproteinase (ADAM)-15 is expressed by the ECs and binds to platelets via GPIIb-IIIa, inducing their activation (14). In vitro studies have demonstrated platelet adhesion to the nonactivated endothelium (11); in another study this linkage was produced under shearing conditions (15). On the other hand, it was demonstrated that ADAM-15 was expressed by the ECs and, by binding to the GPIIb-IIIa complex through its RGD sequence, activated platelets and degraded proteins of the endothelial matrix. This double function of ADAMS-15 could facilitate a rupture of the fibrin layer of the atherosclerotic plaque and accelerate the formation of platelet thrombi (14).

Once platelets are activated, they release adhesive and pro-inflammatory molecules from $\alpha$ granules, which alter the functions of the endothelium (16). For example, platelets store and express CD40L on their surface, releasing this protein to the environment once they are activated so that CD40L is deposited over the endothelium and induces an increase in the expression of molecule adhesion cells (MACs), E-selectin, ICAM-1 and VCAM (17).

Due to their surface expression of CD40L, platelets join the endothelium and are stimulated to release IL- 8 and monocyte chemoattractant protein 1 (MCP-1) (18). This, along with the cytokines released by the endothelium, favours the beginning of leukocyte recruitment at the point of origin of the lesion (1). CD40L also induces the expression and liberation of metalloproteinases, which disintegrate extracellular matrix proteins. These enzymes promote inflammatory response at the site of the injury.

The activated platelets release, among other molecules, IL-1ß, the main EC activator, and promote the release of IL-6 and IL-8 (19), as well as the expression of MACs such as ICAM-1, on the endothelium.

$\mathrm{PF}-4$ is the protein that is released in the highest amount by the activated platelets and causes chemotaxis of monocytes and other leukocytes (20), promoting low-density lipoprotein (LDL) retention by ECs because it inhibits the degradation of the LDL receptor in the endothelium (21).

RANTES chemokine, released from activated T cells, is a chemoattractant for monocytes. In a previous study, it was 


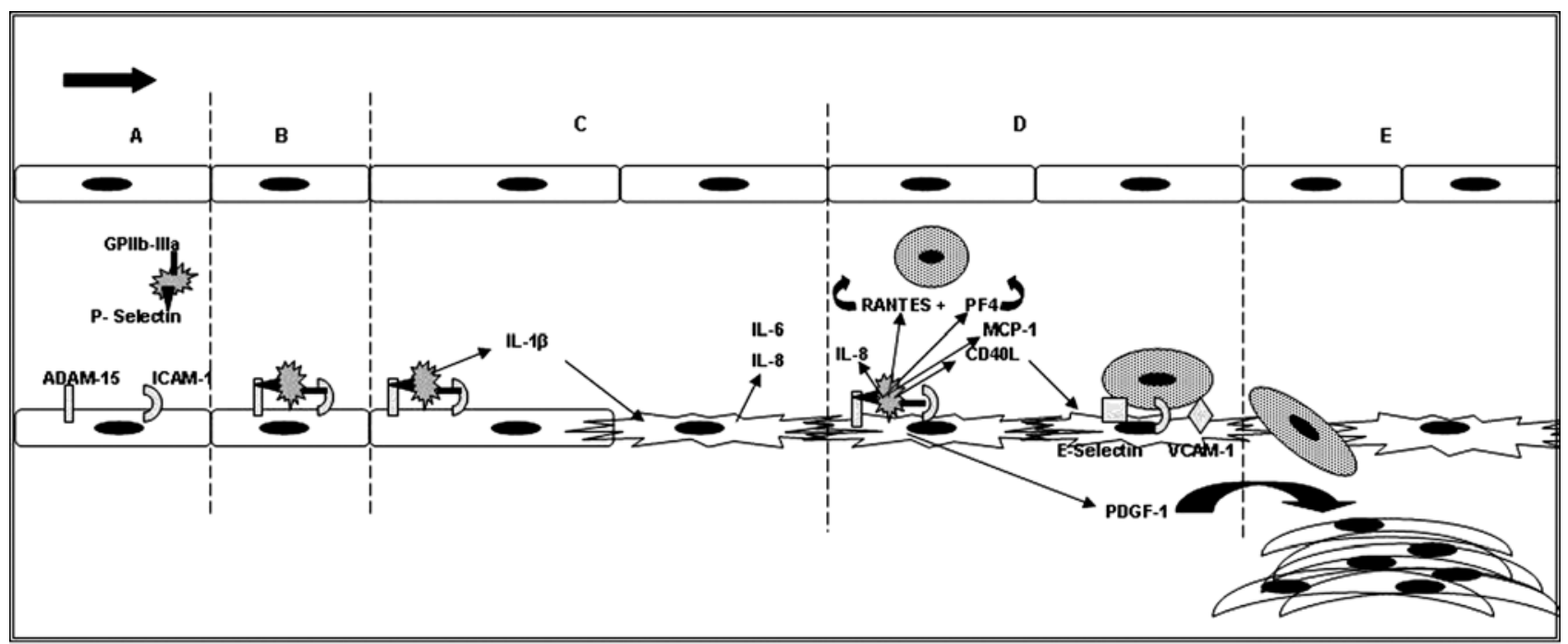

Figure 1. Scheme summarizing the participation of platelets in the process of atherosclerosis. (a) Circulation of activated platelets; (b) the link to the endothelium with activated platelets; (c) activation of endothelial cells; (d) platelet secretion of cytokines and recruiting of leukocytes; and (e) proliferation of smooth muscular cells.

shown that RANTES and PF-4 have heterophilic actions in which PF-4 requires the structural modifications suffered by RANTES to amplify the effects of the monocytes (22). On the other hand, RANTES deposits on the endothelium are promoted by the P-selectin of platelets (23).

PDGF released during platelet activation stimulates the proliferation of muscular cells and produces hyperplasia of the inner section of the vascular wall. It also has a chemotactic function for monocytes, acting as an amplifier of the inflammatory response (24). ENA-78, a chemokine liberated during platelet aggregation, plays a role in monocyte recruitment to the site of injury (25).

In summary, there is experimental evidence to suggest that the circulation of activated platelets initiates the atherosclerotic process through their interaction with intact ECs.

Resting platelets and activated EC. The vascular endothelium is recognized not only as a physical barrier between the blood and tissues, but also as a strategically located organ that possesses numerous paracrine and endocrinal functions. Under physiological conditions, it is capable of exercising important regulatory functions such as vascular tone control, leukocyte adhesion, smooth muscular cell growth and platelet aggregation (26). Diverse factors can alter this balance and cause endothelial dysfunction (25), bringing about a decrease in the capacity for vasodilatation and establishing a proinflammatory and pro-thrombotic state (27).

These alterations of the endothelium can begin at a very young age, thus initiating the slow and progressive development of the lesion. In order for an atheromatosis lesion to begin forming, it is generally necessary for endothelial dysfunction to occur. The first evidence of this is the decrease in nitric oxide synthesis (NO), which provokes alterations in the regulatory functions of the endothelium, such as a decrease in vasodilatadory and anti-thrombotic functions and an increase in permeability and endothelial adhesion (28). Leukocyte adhesion at the site of the lesion represents the earliest histological evidence of plaque development (29).
It has been demonstrated that platelets can roll over the endothelium without being activated, a process which is mediated by endothelial P-selectin (11). Normally, the endothelium is a non-thrombogenic surface. However, during EC apoptosis, pro-adhesive processes are initiated that may allow for the joining of non-activated platelets (15). Thus, activated or dysfunctional endothelium is a determining factor in platelet adhesion.

Hypercholesterolemia promotes endothelial dysfunction by reducing the production of NO (30), thus favouring leukocytary adhesion. When NO decreases, it creates an oxidative environment that oxidizes LDL in transit from the plasma to the site of the endothelial lesion. There, the presence of lisophosphatidylcholin induces the expression of VCAM-1 in the ECs (31).

Under normal conditions, resting platelets do not adhere to the endothelium. However, when they are activated by inflammatory processes or by arterial hypertension, an adhesion surface for platelets can be generated $(32,33)$. It has also been proposed, due to the fact that the endothelium loses certain membrane components and displays anionic phospholipids, that the apoptosis of endothelial cells might play a role (32). P-selectin and E-selectin have been proposed as possible elements participating in EC-platelet interactions $(15,34)$. However, since in other studies the blocking of these receptors did not inhibit platelet linking to the endothelium, it has been postulated that 31 -integrin is a possible receptor (35).

Platelet activation and subsequent aggregation to the altered zones of the vascular walls represents one of the first events in the origin of atherosclerotic lesions (16). In Fig. 1, the participation of the principal molecules associated with the platelets and ECs are summarized.

Microparticles and atherosclerosis. Besides the adhesion and release of cytokines, another phenomenon which accompanies cellular activation is the microvesiculation of the membrane with the generation of microparticles (MPs). MPs are the result of an exocytose process that transports PS from inside the 
cellular membrane to its outside, followed by its breakage, which leads to MP formation and later release. MPs are $0.1 \mu \mathrm{m}$ in size and express PS on their surface. Some antigenic markers from the original cell (36) and, in some cases, the presence of TF have been demonstrated (37). MPs can be released from different types of cells, standing out platelet MPs (PMPs) (38) and endothelial MPs (EMPs) (36).

PMPs are heterogeneous in size and are of lipoprotein composition. They are made up of cytoplasmatic components, anionic phospholipids that contain pro-coagulant potential, platelet membrane glycoproteins, P-selectin and TF, which might extend the thrombogenic process through their interaction with platelets or pro-inflammatory cells (39).

MPs generated in vitro from activated platelets or leukocytes stimulate the ECs in cultures producing prostacyclins and cytokines. Circulating MPs from patients with AIM cause severe endothelial dysfunction in healthy blood vessels when decreasing the NO endothelial path, but do not affect any changes in the expression of NO synthetase (40).

In vitro, EMPs adhere to monocytes, increasing their procoagulant activity. Since MPs express PS and TF (41), EMPmonocyte complexes have been found and have been described as inflammatory disease (42). EMPs have been used as markers for endothelial dysfunction as, according to their phenotype, it is possible to distinguish whether they are derived from activated or apoptotic endothelial cells.

High plasmatic concentrations of EMPs have been found in patients with AIM (40), AHT (43) and DM (44).

It has been suggested that an increase in EMPs and PMPs plays a role in vascular damage. Using a laser to induce endothelial injury in animal models, TF accumulation in the development of in vivo thrombi was found to be dependent on EMPs that carry PSG1-1 during leukocyte rolling, though it is also aided by the linkage of E-selectin during the rolling, and by the migration of in vivo lymphocytes and P-selectin (45).

In diabetic patients, the increase in EC apoptosis and extensive platelet and monocyte activation may contribute to the acceleration of atherothrombosis. In these patients, an increase in the plasmatic levels of EMPs, PMPs and MPs of monocytes has been found, which favours the development of a pro-thrombotic, pro-adhesive and pro-inflammatory condition (46).

Platelet activation releases PMPs that might activate other ECs and leukocytes by means of the release of araquidonic acid and other molecules (47). On the other hand, EMPs might adhere to the platelets, forming EMP-platelet aggregates (47).

The circulation of PMPs can act as an alternative route for the release of RANTES, thus promoting the development of atherosclerotic lesions (48).

Antiplatelet and atherothrombosis drugs. The abovementioned results indicate that platelets participate not only in arterial thrombosis, but also in the initial development of atherogenesis. These findings have motivated the use of platelet anti-aggregant drugs, with the purpose of restricting the development of atherosclerosis. Clopidogrel is an oral platelet anti-aggregant drug which belongs to the thienopyridine family. It selectively and irreversibly inhibits platelet aggregation by preventing the linkage of ADP to its platelet receptors $\mathrm{P}_{2} \mathrm{Y}_{1}$ and $\mathrm{P} 2 \mathrm{Y}_{12}$. In this way, it alters the activation of the complex GPIIb/IIIa and, therefore, the linkage to fibrinogen, inhibiting platelet aggregation (49). Clopidogrel has been widely used to prevent atherosclerotic episodes in patients with a history of AMI or EVA; the drug offers benefits in secondary prevention for these patients (50). The CAPRIE study showed that clopidogrel was more effective than aspirin in reducing the number of ischemic events in high risk individuals (51).

This medicine has been shown to be an efficient antiaggregant of platelets, both in vitro and in vivo (52). In rabbits fed a lipid-rich diet, it was demonstrated that the combination of clopidogrel and aspirin more effectively decreased atherosclerotic lesions of the aorta artery, as well as decreasing concentrations of C-reactive protein (CRP), than each drug did on its own (53). Dogs fed a diet rich in lipids, to whom a combination of clopidogrel, ridogrel and ketanserin was administered, showed an inverse relation between blood flow velocity and platelet inhibition, as well as having a reduced ratio between their medium and intimal arterial layers as compared to the control group (54). Patients with CVD presented exaggerated formation of leukocyte-platelet aggregation. When treated with clopidogrel, they manifested decreased expression of such formation, as well as showing a decrease in the expression of P-selectin (55) and CD40L (56). On the other hand, in patients with a history of ischemic diseases, clopidrogel was shown to be more effective than the ingestion of aspirin in reducing the production of new ischemic events or vascular death (57), as was the case in patients with unstable angina (58).

The use of clopidogrel for five weeks in patients with coronary artery disease resulted in a decrease in the markers of oxidative stress, such as CRP, CD40L and RANTES, as well as in an improvement in the bioavailability of $\mathrm{NO}$ at the endothelial level (59).

\section{Conclusion}

The scientific evidence reported in this review suggests that platelets not only participate in primary and secondary hemostasis, but that they also play a role in the initial stages of the development of atherosclerotic lesions. This role, by means of the secretion of chemokines which promote the expression of MACs over the endothelium, involves the recruitment of inflammatory cells and the stimulation of the activation of other platelets. These findings are supported by the fact that the inhibition of platelet function obtained through the use of drugs such as clopidogrel decreases inflammatory response (in vivo and in vitro) as well as slowing the progression of atherosclerotic lesions.

\section{References}

1. World Health Organization: Informe sobre la salud en el mundo. Technical Report Series ISBN 924 356207X. WHO, Geneva, 2002.

2. Willeit J and Kiechl S: Biology of arterial atheroma. Cerebrovasc Dis 10 (Suppl 5): 1-8, 2000.

3. Vaina $S$ and Stefanadis C: Detection of the vulnerable coronary atheromatous plaque. Where are we now? Int J Cardiovasc Intervent 7: 75-87, 2005.

4. Andrews RK and Berndt MC: Platelet physiology and thrombosis. Thromb Res 114: 447-453, 2004. 
5. Santos M, Aranda E, Vallés J and Palomo I: Hemostasia primaria. In: Hematología: Fisiopatología y Diagnóstico. Palomo I, Pereira J and Palma J (eds). Universidad de Talca, Talca, pp459-492, 2005.

6. Altman R, Berg G and Berman S: Trombosis: Fisiología, mecanismos de enfermedad y tratamiento. In: Trombosis: Fisiopatología, Mecanismos de enfermedad y Tratamiento. Altman R (ed). Akadia, pp3-22, 2005

7. Zhao J, Zhou Q, Wiedmer T and Sims PJ: Level of expression of phospholipid scramblase regulates induced movement of phosphatidylserine to the cell surface. J Biol Chem 273: 6603-6606, 1998.

8. Panes O, Matus V, Saez CG, Quiroga T, Pereira J and Mezzano D: Human platelets synthesize and express functional tissue factor Blood 109: 5242-5250, 2007.

9. Massberg S, Schulz C and Gawaz M: Role of platelets in the pathophysiology of acute coronary syndrome. Semin Vasc Med 3: $147-162,2003$.

10. Siegel-Axel D, Langer H, Lindemann S and Gawaz M: Role of platelets in atherosclerosis and inflammation. Med Klin (Munich) 101: 467-475, 2006.

11. Bombeli T, Schwartz BR and Harlan JM: Adhesion of activated platelets to endothelial cells: evidence for a GPIIb-IIIadependent bridging mechanism and novel roles for endothelial intercellular adhesion molecule 1 (ICAM-1), alphavbeta3 integrin, and GPIbalpha. J Exp Med 187: 329-339, 1998

12. Huo Y, Schober A, Forlow SB, et al: Circulating activated platelets exacerbate atherosclerosis in mice deficient in apolipoprotein E. Nat Med 9: 61-67, 2003.

13. Blann AD, Nadar SK and Lip GY: The adhesion molecule P-selectin and cardiovascular disease. Eur Heart J 24: 2166-2179, 2003.

14. Langer H, May AE, Bultmann A and Gawaz M: ADAM 15 is an adhesion receptor for platelet GPIIb-IIIa and induces platelet activation. Thromb Haemost 94: 555-561, 2005.

15. Frenette PS, Johnson RC, Hynes RO and Wagner DD: Platelets roll on stimulated endothelium in vivo: an interaction mediated by endothelial P-selectin. Proc Natl Acad Sci USA 92: 7450-7454, 1995.

16. Gawaz M, Langer $\mathrm{H}$ and May AE: Platelets in inflammation and atherogenesis. J Clin Invest 115: 3378-3384, 2005.

17. Henn V, Slupsky JR, Grafe M, et al: CD40 ligand on activated platelets triggers an inflammatory reaction of endothelial cells. Nature 391: 591-594, 1998.

18. Gawaz M, Brand K, Dickfeld T, et al: Platelets induce alterations of chemotactic and adhesive properties of endothelial cells mediated through an interleukin-1-dependent mechanism. Implications for atherogenesis. Atherosclerosis 148: 75-85, 2000.

19. Hawrylowicz CM, Howells GL and Feldmann M: Plateletderived interleukin 1 induces human endothelial adhesion molecule expression and cytokine production. J Exp Med 174: 785-790, 1991.

20. Kaplanski G, Porat R, Aiura K, Erban JK, Gelfand JA and Dinarello CA: Activated platelets induce endothelial secretion of interleukin-8 in vitro via an interleukin-1-mediated event. Blood 81: 2492-2495, 1993.

21. Scheuerer B, Ernst M, Durrbaum-Landmann I, et al: The CXCchemokine platelet factor 4 promotes monocyte survival and induces monocyte differentiation into macrophages. Blood 95: 1158-1166, 2000.

22. Nassar T, Sachais BS, Akkawi S, et al: Platelet factor 4 enhances the binding of oxidized low-density lipoprotein to vascular wall cells. J Biol Chem 278: 6187-6193, 2003.

23. Von Hundelshausen P, Koenen RR, Sack M, et al: Heterophilic interactions of platelet factor 4 and RANTES promote monocyte arrest on endothelium. Blood 105: 924-930, 2005.

24. Schober A, Manka D, von Hundelshausen P, et al: Deposition of platelet RANTES triggering monocyte recruitment requires $\mathrm{P}$-selectin and is involved in neointima formation after arterial injury. Circulation 106: 1523-1529, 2002.

25. Baltus T, von Hundelshausen P, Mause SF, Buhre W, Rossaint R and Weber C: Differential and additive effects of platelet-derived chemokines on monocyte arrest on inflamed endothelium under flow conditions. J Leukoc Biol 78: 435-441, 2005.

26. Tang J, Kozaki K, Farr AG, et al: The absence of plateletderived growth factor-B in circulating cells promotes immune and inflammatory responses in atherosclerosis-prone $\mathrm{ApoE}^{-/}$ mice. Am J Pathol 167: 901-912, 2005.

27. Rojas A and Morales MA: Advanced glycation and endothelial functions: a link towards vascular complications in diabetes. Life Sci 76: 715-730, 2004.
28. Verma S, Buchanan MR and Anderson TJ: Endothelial function testing as a biomarker of vascular disease. Circulation 108: 2054-2059, 2003.

29. Rojas A, Romay S, Gonzalez D, Herrera B, Delgado R and Otero K: Regulation of endothelial nitric oxide synthase expression by albumin-derived advanced glycosylation end products. Circ Res 86: E50-E54, 2000.

30. Willerson JT and Kereiakes DJ: Endothelial dysfunction. Circulation 108: 2060-2061, 2003.

31. Cybulsky MI, Lichtman AH, Hajra L and Iiyama K: Leukocyte adhesion molecules in atherogenesis. Clin Chim Acta 286: 207-218, 1999.

32. Landmesser U, Hornig B and Drexler H: Endothelial dysfunction in hypercholesterolemia: mechanisms, pathophysiological importance, and therapeutic interventions. Semin Thromb Hemost 26: 529-537, 2000.

33. Iiyama K, Hajra L, Iiyama M, et al: Patterns of vascular cell adhesion molecule- 1 and intercellular adhesion molecule-1 expression in rabbit and mouse atherosclerotic lesions and at sites predisposed to lesion formation. Circ Res 85: 199-207, 1999.

34. Bombeli T, Karsan A, Tait JF and Harlan JM: Apoptotic vascular endothelial cells become procoagulant. Blood 89: 2429-2442, 1997.

35. Marcus AJ and Safier LB: Thromboregulation: multicellular modulation of platelet reactivity in hemostasis and thrombosis. FASEB J 7: 516-522, 1993

36. Dong ZM, Chapman SM, Brown AA, Frenette PS, Hynes RO and Wagner DD: The combined role of P- and E-selectins in atherosclerosis. J Clin Invest 102: 145-152, 1998.

37. Bombeli T, Schwartz BR and Harlan JM: Endothelial cells undergoing apoptosis become proadhesive for nonactivated platelets. Blood 93: 3831-3838, 1999.

38. Horstman LL, Jy W, Jimenez JJ, Bidot C and Ahn YS: New horizons in the analysis of circulating cell-derived microparticles. Keio J Med 53: 210-230, 2004

39. Tedgui A and Mallat Z: Smooth muscle cells: another source of tissue factor-containing microparticles in atherothrombosis? Circ Res 87: 81-82, 2000

40. Nomura S: Platelet activation marker. Rinsho Byori 51: 1096-1101, 2003

41. Brodsky SV, Malinowski K, Golightly M, Jesty J and Goligorsky MS: Plasminogen activator inhibitor-1 promotes formation of endothelial microparticles with procoagulant potential. Circulation 106: 2372-2378, 2002.

42. Boulanger CM, Scoazec A, Ebrahimian T, et al: Circulating microparticles from patients with myocardial infarction cause endothelial dysfunction. Circulation 104: 2649-2652, 2001.

43. Mallat Z, Hugel B, Ohan J, Leseche G, Freyssinet JM and Tedgui A: Shed membrane microparticles with procoagulant potential in human atherosclerotic plaques: a role for apoptosis in plaque thrombogenicity. Circulation 99: 348-353, 1999.

44. Sabatier F, Roux V, Anfosso F, Camoin L, Sampol J and DignatGeorge F: Interaction of endothelial microparticles with monocytic cells in vitro induces tissue factor-dependent procoagulant activity. Blood 99: 3962-3970, 2002.

45. Preston RA, Jy W, Jimenez JJ, et al: Effects of severe hypertension on endothelial and platelet microparticles. Hypertension 41: 211-217, 2003.

46. Koga H, Sugiyama S, Kugiyama K, et al: Elevated levels of remnant lipoproteins are associated with plasma platelet microparticles in patients with type-2 diabetes mellitus without obstructive coronary artery disease. Eur Heart J 27: 817-823, 2006.

47. Falati S, Liu Q, Gross P, et al: Accumulation of tissue factor into developing thrombi in vivo is dependent upon microparticle P-selectin glycoprotein ligand 1 and platelet P-selectin. J Exp Med 197: 1585-1598, 2003.

48. Nomura S, Suzuki M, Katsura K, et al: Platelet-derived microparticles may influence the development of atherosclerosis in diabetes mellitus. Atherosclerosis 116: 235-240, 1995.

49. Chirinos JA, Heresi GA, Velasquez H, et al: Elevation of endothelial microparticles, platelets, and leukocyte activation in patients with venous thromboembolism. J Am Coll Cardiol 45: $1467-1471,2005$.

50. Mause SF, von Hundelshausen P, Zernecke A, Koenen RR and Weber C: Platelet microparticles: a transcellular delivery system for RANTES promoting monocyte recruitment on endothelium. Arterioscler Thromb Vasc Biol 25: 1512-1518, 2005.

51. Izaguirre Avila R, De La Pena A, Gonzalez Pacheco H, et al: Efficacy of clopidogrel as ADP-dependent platelet aggregation inhibitor. Study on individuals with coronary artery disease. Arch Inst Cardiol Mex 70: 472-480, 2000. 
52. CAPRIE Steering Committee: A randomised, blinded, trial of clopidogrel versus aspirin in patients at risk of ischaemic events (CAPRIE). Lancet 348: 1329-1339, 1996.

53. Easton JD: Evidence with antiplatelet therapy and ADP-receptor antagonists. Cerebrovasc Dis 16 (Suppl 1): 20-26, 2003.

54. Gu Q, Chen JL and Ruan YM: Inhibitory effects of aspirin, clopidogrel, and their combination on the progression of atherosclerosis in rabbits. Zhongguo Yi Xue Ke Xue Yuan Xue Bao 27: 87-91, 2005.

55. Anderson HV, McNatt J, Clubb FJ, et al: Platelet inhibition reduces cyclic flow variations and neointimal proliferation in normal and hypercholesterolemic-atherosclerotic canine coronary arteries. Circulation 104: 2331-2337, 2001.
56. Klinkhardt U, Bauersachs R, Adams J, Graff J, Lindhoff-Last E and Harder S: Clopidogrel but not aspirin reduces P-selectin expression and formation of platelet-leukocyte aggregates in patients with atherosclerotic vascular disease. Clin Pharmacol Ther 73: 232-241, 2003.

57. Graff J, Harder S, Wahl O, Scheuermann EH and Gossmann J: Anti-inflammatory effects of clopidogrel intake in renal transplant patients: effects on platelet-leukocyte interactions, platelet CD40 ligand expression, and proinflammatory biomarkers. Clin Pharmacol Ther 78: 468-476, 2005.

58. Jarvis B and Simpson K: Clopidogrel: a review of its use in the prevention of atherothrombosis. Drugs 60: 347-377, 2000.

59. Jain A, Wadehra V and Timmis AD: Management of stable angina. Postgrad Med J 79: 332-336, 2003. 